\title{
Unilateral external jugular vein fenestration with variant anatomy of the retromandibular and facial vein
}

\author{
Sharmila Bhanu Ponnambalam, Devi Sankar Karuppiah \\ Department of Anatomy, Narayana Medical College, Nellore, India
}

\begin{abstract}
External jugular vein (EJV), a significant superficial vein draining head and neck (H\&N) region is been increasingly used for cannulation in diverse diagnostic purposes and intravenous therapies. The variant anatomy of formation and draining patterns of EJV and retromandibular veins were reported earlier. In the present case, EJV showed significant variation in one of the male cadaver, during routine $\mathrm{H} \& \mathrm{~N}$ dissection. On the left side, a fenestration of EJV was observed and the transverse cutaneous nerve of the neck was passing through the fenestration of the vein. Anatomical knowledge of EJV and retromandibular vein variations as found in this case is important for surgeons performing microvascular surgeries in H\&N and also to the radiologists during their conventional radiological procedures like angioplasty, catheterization, and at times of hemodialysis in case of renal failure patients.
\end{abstract}

Key words: Vascular fenestration, External jugular vein, Common facial vein, Retromandibular vein

Received August 8, 2019; Revised August 23, 2019; Accepted September 9, 2019

\section{Introduction}

Divergence of the vascular system from its normal pattern is commonly observed in veins than the arteries [1]. Superficial veins of the body such as external jugular vein (EJV) show a considerable variation in its morphology, size, formation, and termination. Fenestration or incomplete divisions of craniocervical blood vessels are reported in the literature. Among the vessels around the head and neck $(\mathrm{H} \& \mathrm{~N})$ regions, the arterial fenestrations are commonly observed than the venous fenestrations [2]. Many H\&N arterial fenestrations have been reported in branches of the circle of Willis (frequently), vertebral artery, and internal carotid artery [3]. Fenestration

\footnotetext{
Corresponding author:

Devi Sankar Karuppiah (it)

Department of Anatomy, Narayana Medical College, Chinthareddy Palem, Nellore, Andhra Pradesh 524002, India

Tel: +91-9490948006, Fax: +91-861-2317962, E-mail: lesanshar@gmail. com
}

of EJV is a very rare incidence unlike the other anomalies of $\mathrm{H} \& \mathrm{~N}$ vasculature. Mostly these fenestrated vessels were not transmitting any structures through it, but very rarely some structures may pass through the venous fenestration [4].

The EJV, one of the main superficial veins of the $H \& N$, drains blood mostly from the scalp and the face. Normally it is formed or begins near the angle of the mandible by the union of the posterior division of the retromandibular vein (RMV) with the posterior auricular vein (PAV). It runs downwards but deep to platysma in the posterior cervical triangle; then it pierces the deep cervical fascia at the posterior border of the sternocleidomastoid muscle. Further, the EJV descends into the inferior part of this triangle and terminates in the subclavian vein [5]. Along its course in the lower half of the posterior triangle, the vein crosses the transverse cervical nerve $\left(C_{2-3}\right)$ superficially. The clinical importance of this vein lies in the fact that it is easier to visualize than the internal jugular vein (IJV) and can give a more reliable estimate of central venous pressure [6].

Another significant variation that has been reported in the 
literature is the variations of RMV and draining pattern of the facial vein (FV) with undivided RMV continuing as EJV and directly draining into EJV or FV [7].

Here we present a rare variation of EJV with a large fenestration in the neck region with an exceptional finding of transverse cutaneous nerve passing through along with undivided RMV and varying draining pattern of FV.

\section{Case Report}

During posterior cervical triangle dissection for the firstyear medical students, one of the male cadaver aged 52 years showed variations of EJV and RMV on the left side. The EJV showed a vertical fenestration (Fig. 1A), the length of which was found to be $4.8 \mathrm{~cm}$. The fenestration commenced from the level of apex of the parotid gland and extended vertically downwards in the neck. Prior to its termination just above the middle third of the clavicle, the fenestrated EJV joined to form a single trunk pierced the deep cervical fascia and drained into the subclavian vein. No dilatations were found proximal and distal to fenestration. Branches of the transverse cervical nerve were found passing through this fenestration (Fig. 1A). The left RMV directly continued as the EJV and the classical termination of RMV into anterior and posterior divisions was completely absent. Near the apex of the parotid gland, behind and inferior to the angle of mandible, the PAV drained into the RMV. A long communicating vein between the RMV and anterior jugular vein was found running on the anterior surface of the sternocleidomastoid muscle. The left FV was found running posteroinferior at the anterior border of masseter and drained directly into the left IJV, deep to the sternocleidomastoid muscle (Fig. 1B). No such variations were found on right side $\mathrm{H} \& \mathrm{~N}$ region.

\section{Discussion}

The awareness of anomalous venous patterns in the region of $\mathrm{H} \& \mathrm{~N}$ is essential for the surgeons performing intraoperative microvascular surgeries to avoid unnecessary bleeding. Among the superficial veins of $H \& N$, the EJV is reported to exemplify numerous variations that affect its size, form, course, tributaries, drainage, and termination [8].

Among the veins of $\mathrm{H} \& \mathrm{~N}$, IJV fenestration occurs in $0.4 \%$ of the population [9] and only a very few cases been reported in the literature so far [10].

Superficial venous fenestrations are rare and EJV fenestration is exclusively a very rare anomaly found in the H\&N. In 2010, Choudhary et al. [11] reported that the EJV was found to be split into two channels and got reunited at its junction with the FV but no structures were passing through it.

In the present case, left side EJV showed a large central fenestration which rejoined in the lower part to form a single trunk. The significant feature found in this anomaly was the transverse cervical nerve was passing through this fenestra-
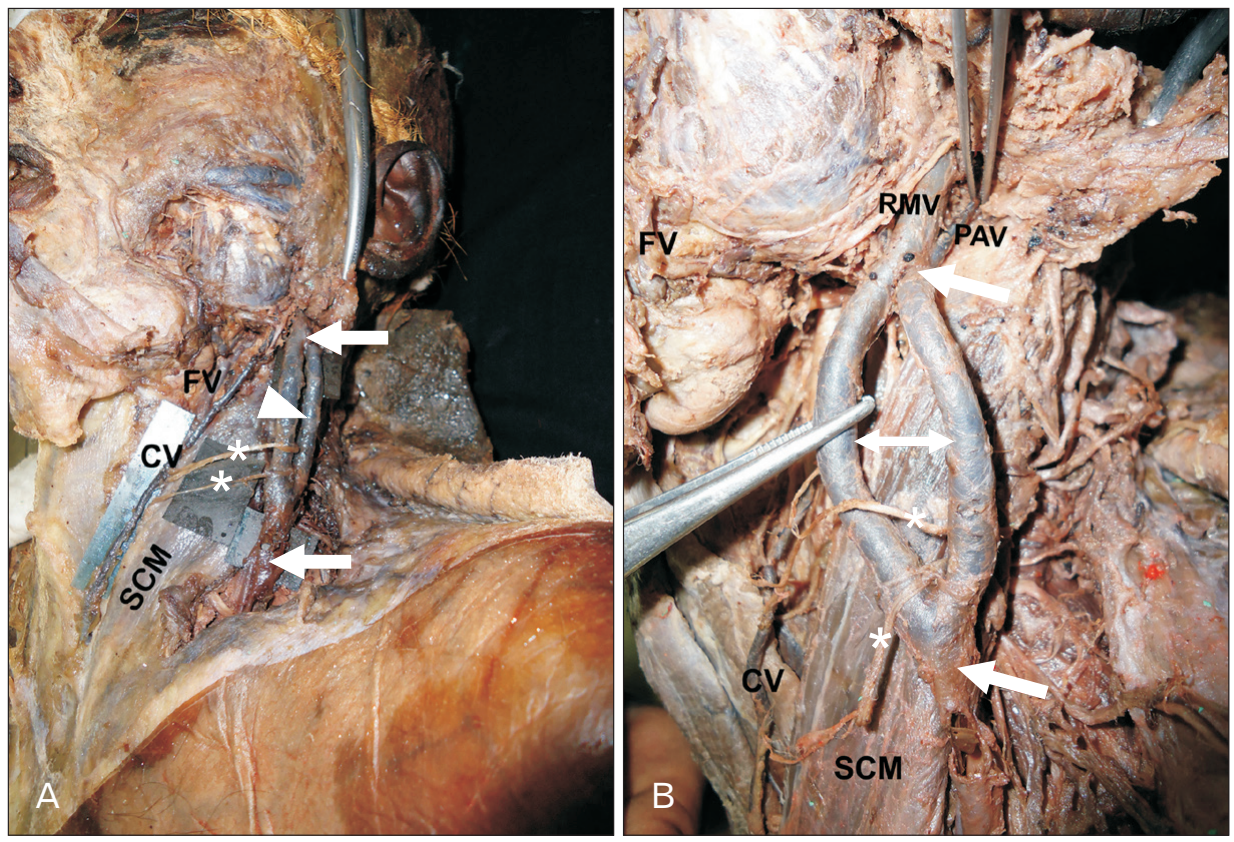

Fig. 1. (A) Dissection showing the fenestration (arrowhead) of the left external jugular vein (arrows). The asterisks indicate transverse cervical nerves. (B) Large fenestration (double-sided arrow) of left external jugular vein (arrows). The asterisks indicate transverse cervical nerves. CV, communicating vein between left side retromandibular and anterior jugular veins; FV, facial vein; PAV, posterior auricular vein; RMV, retromandibular vein; SCM, sternocleidomastoid muscle. 
tion. Coinciding with the present study, one case was reported with supraclavicular nerve passing through a partial duplication of the EJV in which the vein was forming a small venous ring towards its termination [12].

Duplication or fenestration of the EJV can be supported in the developmental background. But there is no clear evidence or hypothetical views for the formation of the fenestrated EJV till date have been found in the literature. According to the embryological delineation of the vascular system of $\mathrm{H} \& \mathrm{~N}$, the proximal end of the primitive cephalic vein forms EJV. The venous plexus surrounding the tissues of the neck and make an anterior connection with FV and posteriorly with the RMV [13]. During further development, the anterior connection disappears and the FV joins with an anterior division of RMV drains into the IJV (Fig. 2A). The RMV develops from the temporal region and joins the linguofacial vein to form common FV (CFV) which drains into the pre-cardinal vein, the future IJV.

The fenestration in the present case may be due to the persistent anterior connections of the EJV. The anterior division of the RMV would have joined persisted anterior connection and resulted in the fenestration of the EJV on the left side (Fig. 2B). While extending the formation of venous channels surrounding the $\mathrm{H} \& \mathrm{~N}$ would have been the cause of transverse cutaneous nerve passing through the fenestration.

The customary anterior and posterior divisions of RMV was absent in the present case, instead, it joined PAV to form
EJV. The CFV was absent which showed that RMV failed to establish the anterior connection with the FV. Similar cases of absence of CFV and FV draining directly into IJV was reported by González-Garcia et al. [9]. The cases of FV draining into a superficial temporal vein with an undivided RMV [14] and termination of FV into the EJV have been reported in the literature [15].

The EJV is a main peripheral vein that can be accessed for administering non-sclerosing agents. Knowledge of fenestration of EJV and variations in the veins of the face and neck are of immense important both clinically and surgically. Awareness of these types of variations helps to avoid radiologic misinterpretations or misidentifications of the veins of the neck during the conventional radiographic procedures like angiography and catheterization or hemodialysis in renal failure patients. In cases of fenestrated EJV with the structures passing through it like the transverse cutaneous nerve of the neck makes it a rare variation where care should be taken during block dissections of the neck and reconstructive surgeries of brachial plexus. A sound anatomic knowledge of variations in the formation and draining patterns of the FV and its tributaries are essential for the microvascular surgical procedures to avoid errors leading to excessive bleeding. Expertise acquaintance in the venous pattern of $\mathrm{H} \& \mathrm{~N}$ for the medical practitioners dealing with these is necessary in order to avoid major and minor complications in any type of procedures in these regions.
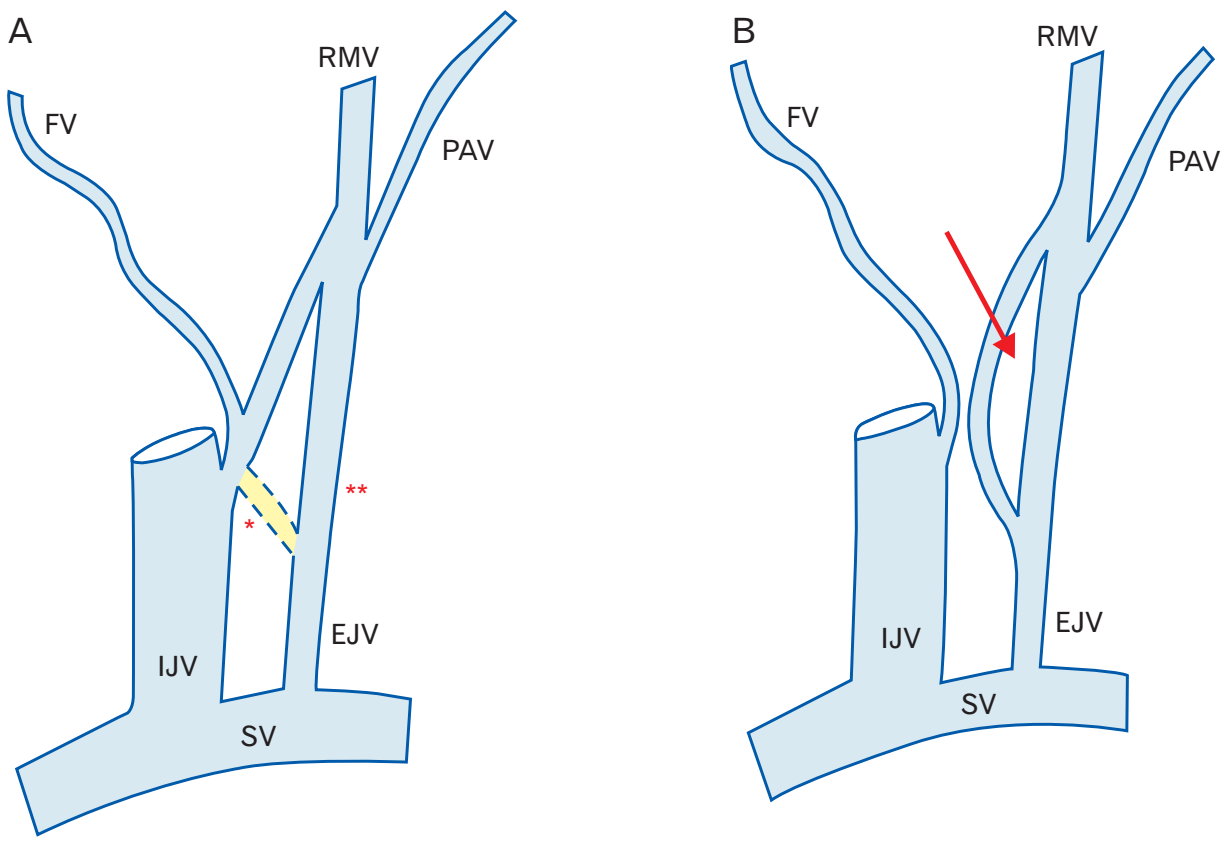

Fig. 2. Schematic representation of the embryonic formation of the normal (A) and fenestrated external jugular vein (B). Single and double asterisk indicate persisting embryonic anterior and posterior connections of EJV respectively, thus forms the fenestration (arrow). EJV, external jugular vein; FV, facial vein; IJV, internal jugular vein; PAV, posterior auricular vein; RMV, retromandibular vein; SV, subclavian vein. 


\section{ORCID}

Sharmila Bhanu Ponnambalam:

https://orcid.org/0000-0002-9191-6131

Devi Sankar Karuppiah:

https://orcid.org/0000-0001-7025-723X

\section{Author Contributions}

Conceptualization: SBP. Data acquisition: SBP. Data analysis or interpretation: DSK. Drafting of the manuscript: DSK. Critical revision of the manuscript: SBP. Approval of the final version of the manuscript: all authors.

\section{Conflicts of Interest}

No potential conflict of interest relevant to this article was reported.

\section{References}

1. Hollinshead WH. Anatomy for surgeons. Vol. 1. The head and neck. 3rd ed. Jagerstown: Harper \& Row; 1982.

2. Towbin AJ, Kanal E. A review of two cases of fenestrated internal jugular veins as seen by CT angiography. AJNR Am J Neuroradiol 2004;25:1433-4.

3. Sanders WP, Sorek PA, Mehta BA. Fenestration of intracranial arteries with special attention to associated aneurysms and other anomalies. AJNR Am J Neuroradiol 1993;14:675-80.

4. Comert E, Comert A. External jugular vein duplication. J Craniofac Surg 2009;20:2173-4.

5. Standring S. Gray's anatomy: the anatomical basis of clinical practice. 41st ed. Philadelphia, PA: Elsevier; 2016. p.459, 505.

6. Nagata T, Masumoto K, Watanabe Y, Katou F. End-to-side anastomosis to the external jugular vein: preservation of external jugular vein blood flow. Br J Oral Maxillofac Surg 2012;50:e31-2.

7. Bertha A, Rabi S. Anatomical variations in termination of common facial vein. J Clin Diagn Res 2011;5:24-7.

8. Silva MR, Henriques JG, Silva JH, Camargos VR, Moreira PR. Venous arrangement of the head and neck in humans: anatomic variability and its clinical inferences. J Morphol Sci 2016;33:22-8.

9. González-García R, Román-Romero L, de la Plata MM. The rare phenomenon of internal jugular vein duplication. Otolaryngol Head Neck Surg 2007;137:847-8.

10. Devi Sankar K, Sharmila Bhanu P. Variant anatomy of fenestrated internal jugular vein with unusual retromandibular and facial veins. Int J Anat Var 2011;4:144-6.

11. Choudhary S, Sharma AK, Singh H. Undivided retromandibular vein continuing as external jugular vein with facial vein draining into it: an anatomical variation. JK Sci 2010;12:203-4.

12. Singla RK, Singla S, Sachdeva K. Partial duplication of external jugular vein forming a venous ring round supraclavicular nerve: a case report. J Clin Diagn Res 2011;5:859-61.

13. Patil J, Kumar N, Swamy RS, D’Souza MR, Guru A, Nayak SB. Absence of retromandibular vein associated with atypical formation of external jugular vein in the parotid region. Anat Cell Biol 2014;47:135-7.

14. Peuker ET, Fischer G, Filler TJ. Facial vein terminating in the superficial temporal vein: a case report. J Anat 2001;198(Pt 4):50910.

15. D'Silva SS, Pulakunta T, Potu BK. Termination of the facial vein into the external jugular vein: an anatomical variation. J Vasc Bras 2008;7:174-5. 